Special Issue on Bone Disease Mechanisms:

\title{
Periostin action in bone
}

Nicolas Bonnet, Patrick Garnero, Serge Ferrari

Division of Bone Diseases, Department of Internal Medicine Specialties, Geneva University Hospitals \& Faculty of Medicine, Geneva 14, Switzerland.

Bonnet Nicolas $(\mathrm{PhD})$

Geneva University Hospital \& Faculty of Medicine

Department of Internal Medicine Specialties

Division of Bone Diseases

64 Av de la Roseraie

CH-1205 Geneva 14

Switzerland

Tel: 0041/ 223829968

Fax: 0041/ 223829973 


\begin{abstract}
Periostin is a highly conserved matricellular protein that shares close homology with the insect cell adhesion molecule fasciclin 1. Periostin is expressed in a broad range of tissues including the skeleton, where it serves both as a structural molecule of the bone matrix and a signaling molecule through integrin receptors and Wnt-beta-catenin pathways whereby it stimulates osteoblast functions and bone formation. The development of periostin null mice has allowed to elucidate the crucial role of periostin on dentinogenesis and osteogenesis, as well as on the skeletal response to mechanical loading and parathyroid hormone.
\end{abstract}




\section{Introduction}

The processes that control bone homeostasis in response to hormonal influences, mechanical loading and tissue injury are complex and involve a myriad of growth factors, cytokines and other molecules expressed in the bone environment. The ultimate result of these physiological processes, namely the maintenance, gain or loss of bone mass and strength depends on the degree of coupling between osteoblasts, - the bone forming cell-, and osteoclasts, -the bone resorbing cell-, and their coordination by osteocytes, the end-product of osteoblasts differentiation. The latter are increasingly considered as the "astrocytes" of bone, which not only control bone modeling, i.e. the activity of bone forming lining cells without prior resorption, but also bone remodeling, i.e. bone formation following bone resorption (1). As the cellular effector of the skeletal mechanostat, osteocytes mainly repress bone formation by the production of sclerostin, a main inhibitor of Wnt-beta-catenin signaling in osteoblasts (2). This signaling pathway has recently been found to also play a role in osteocytes themselves, notably to control their expression of OPG, a main inhibitor of bone resorption (3). More recently, another molecule primarily expressed in bone by osteocytes, and capable to modulate Wnt-beta-catenin signaling has emerged as another key player in bone homeostasis: periostin, which pleiotropic functions in bone, regulation and potential use as a novel (cortical) bone marker are reviewed here.

\section{Expression, structure and function of periostin}

Periostin (Postn) is a $90 \mathrm{kD}$ extracellular matrix protein of 836 aa (in humans), originally named osteoblast specific factor 2 (OSF-2) when it was first cloned from a cDNA library prepared from the mouse osteoblastic cell line MC3T3-E1 (4). Although the current denomination derives from its expression in the periosteum of long bones, periostin is broadly expressed, with highest levels in the aorta, stomach, lower gastrointestinal tract, placenta, 
uterus, thyroid tissue and breast. It is particularly expressed during ontogenesis and in adult connective tissues submitted to mechanical stimulation (stretch), such as heart valves, skin, periodontal ligaments, tendons and bones. Postn gene expression is up-regulated by plateletderived growth factor (PDGF) and basic fibroblast growth factors (FGF) in cancer lines through different pathways including PI3K and p38MAPK. In bone, Postn is transcriptionally regulated by Twist, RUNX2, and C-Fos/AP1. Its expression levels are maximal in the periosteum and osteocytes (see below) and further controlled by mechanical stimuli, hormones (PTH), growth factors (TGF- $\beta$, BMP2) and cytokines (TNF- $\alpha$, IL-4, IL-13, and likely PDGF), all known to have important roles in the determination and/or regulation of bone homeostasis (5).

Periostin is constituted of a signal sequence, followed by an Emilin-like domain rich in cysteine, four repeated fasiclin 1 , and a $\mathrm{C}$ terminal variable domain which gives 7 splice variants human isoforms. Periostin sequence is highly conserved during evolution, with 99.9\% identity between human and gorilla, 96\% with dogs and 90\% identity with mice. However Postn can be modified post-translationally by a vitamin K dependent enzyme which changes glutamic residues (Glu) to $\gamma$-carboxyglutamate (Gla) (6), because Postn contains 28 potential sites of carboxylation. Whether this gamma carboxylation is present in bone and alters the function of Postn, as it is the case for osteocalcin, is currently unknown.

Periostin has at least two major functions, one in fibrillogenesis which occurs in the matrix and another in cell migration (7). Thus, in bone, periostin enhances the proteolytic activation of lysyl oxidase which is required for collagen cross-linking. This activity is accomplished by the ability of periostin to directly interact with type I collagen, fibronectin, Notch1, tenascin-C and BMP-1. In addition, periostin activates integrin-mediated signaling (see below), thereby promoting cell adhesion and motility by activation of the actin/myosin contractile machinery $(8,9)$ (Figure 1). 


\section{Periostin-activated signaling pathways}

Current knowledge about periostin signaling mainly comes from studies aiming to elucidate its role in tumorigenesis and the interaction between cancer cells and their metastatic niche (10). Periostin interacts primarily with integrin receptors $\alpha \mathrm{V} \beta 3 \& \alpha \mathrm{V} \beta 5$ which initiate a crosstalk with receptor tyrosine kinases (RTKs), such as EGFR and VEGF (11) (Figure 1). Periostin binding to integrins activates AKT/PKB and the FAK-mediated signaling pathways, while inhibiting GSK3$\beta$, -a main regulator of the Wnt- $\beta$-catenin signaling pathway-, through PiP3 and/or AKT (12). Periostin is also capable to indirectly decrease $\beta$-catenin degradation by blocking PTEN, an activator of $\beta$-catenin degradation (13). Thus periostin was shown to activate Wnt- $\beta$ catenin signaling in primary osteoblastic cell cultures and in TOPGAL reporter mice which uses a ß-galactosidase reporter gene (LacZ) under the control of multimerized TCF binding sites (REF) (Figure 1). Moreover in epithelial cells periostin was shown to activate mTOR signaling through phosphorylation of AKT, which mediates mitogenesis and migration of these cells (14).

\section{Periostin functions in bone cells}

In MC3T3-E11 osteoblastic cells, overexpression of Postn increases cell proliferation and differentiation (15). Consistent with these in vitro observations, injection of an adenovirus overexpressing periostin, increased bone formation index and bone mass in rats (16). Conversely, Postn deletion results in a defective attachment of osteoblasts to the bone matrix, which affects their differentiation into mature osteoblasts as shown by a severe reduction of the expression of type I collagen, osteocalcin, osteopontin and alkaline phosphatase, as well as a decreased mineralization process in vitro $(17,18)$. In the UMR-106 osteosarcoma cell line, periostin inhibits sclerostin gene (Sost) expression, whereas periostin neutralizing 
antibodies markedly reduced Sost inhibition by PTH. Moreover, blocking $\alpha \mathrm{V} \beta 3$ integrin receptors prevented sclerostin inhibition by periostin, indicating that in addition to its effects on osteoblasts, periostin may have autocrine functions on sclerostin-expressing cells (18). Coimmunoprecipitation experiments further suggest that periostin may directly interact with sclerostin to inhibit sclerostin antagonistic activity on WNT signaling and thereby it promotes bone formation by MC3T3 in response to BMP and Wnt ligands (19).

In the IDG-W3 cell line, Postn expression increased with the differentiation of the late osteoblast into osteocytes (Figure 2A). In these cells, Postn levels were stimulated upon exposure to PTH (Figure 2B). Similarly, Postn is rapidly up-regulated in response to PTH in osteocytes in vivo (18). Thus PTH-stimulated Postn expression could regulate Sost and possibly Wnt- $\beta$ catenin regulated genes in osteocytes, such as OPG, by an autocrine mechanism. It remains to be elucidated whether periostin affects other osteocytic cell functions, such as osteocytic osteolysis during lactation, as well as the survival of osteocytes. In this context, it is interesting to note that 3 months administration of high-dose zoledronate decreased the number of osteocytes in both wild type and Postn $\mathrm{KO}$ mice, but increased the number of empty osteocyte lacunae only in the latter, suggesting an anti-apoptotic effect of periostin in osteocytes(20).

Under physiological condition, immunohistochemical staining for periostin in bone does not reveal any significant expression in osteoclasts. However recent in vitro studies suggest that Postn is also expressed at low levels in osteoclasts from mouse long bones differentiated in vitro (21). Whether periostin expressed by osteoclasts represents yet another coupling factor with osteoblasts and/or exerts autocrine functions in these cells (which actually express integrin receptors to which periostin could bind) remains to be elucidated (also see "lessons from Postn KO mice, below). 


\section{Periostin functions in fracture healing}

In rodent models of transverse tibial diaphysis fracture, periostin mRNA and protein levels are rapidly upregulated during fracture healing, particularly in proliferating osteoblast cells of the periosteum as well as in undifferentiated mesenchymal cells. Periostin has been demonstrated to be involved in various phases of bone repair: (1) early on during inflammation and angiogenesis; (2) on the recruitment of osteoprogenitors into the callus; (3) in the early stages of osteoblast differentiation and bone formation. In a calvarial bone defect model, periostin has been shown to increase numbers of CD-31-positive endothelial cells and $\alpha$-SMA-positive arterioles within the defect, indicating an effect on angiogenesis $(22,23)$. The role of periostin in angiogenesis has been confirmed in other tissues, namely keloids (overgrowth of granulation tissue) through the activation of the ERK and focal adhesion kinase pathway, as well as the upregulated expression of VEGF and angiopoietin-1. Indeed there was a good correlation between periostin levels and blood vessel density in keloid tissue $(\mathrm{r}=0.711, \mathrm{p}<0.01)(24)$. To our knowledge this interaction has never been directly investigated in long bones. Considering the important role of vascularization in bone metabolism and bone repair, a better understanding of the interaction between periostin and angiogenesis might expand our knowledge on bone remodeling response to gravity, PTH and fracture repair.

\section{Lessons from the Post KO mice}

\section{Skeletal phenotype of Postn KO mice}

Periostin functions have now been extensively investigated thanks to the development of Postn- deficient mice (25). At first periostin was shown to be expressed in the periodontal ligament, where it acts as a critical regulator of tooth formation and maintenance. Experimental tooth movement has been shown to increase periodontal fibroblast proliferation 
in association with increased periostin mRNA and protein expression (26). Accordingly, Postn-deficient mice show severe alterations in tooth eruption, resulting from a failure to digest collagen fibers in the shear zone of the periodontal ligament (27). As a consequence, the enamel and dentin of the incisors is compressed and disorganized and the jaw bone is altered (25). More specifically, the crestal alveolar bone is decreased, and the periodontal ligament appeared enlarged. Basal bone, which does not belong to the periodontal tissue, was also affected as fibrous areas were increased in Postn KO (20).

Postn KO mice exhibit a low bone mass and cortical architecture, with a reduced cortical bone volume and a decrease in bone strength in young adult mice, which is accentuated with aging $(25,28)$. The features are primarily associated with reduced bone formation, particularly at periosteal surfaces, with a marked decrease in MAR suggesting an effect mainly on the activity of lining cells and cortical expansion. Taken together with the predominant expression of periostin in osteocytes and the periosteum, these observations also suggested a role of periostin in modeling-based responses to mechanical loading (i.e. the mechanostat, see below).

In addition, in Postn KO mice at one year of age, there is evidence of increased intracortical bone remodeling (Figure 3). Postn KO mice did not exhibit changes in TRAP5b activity or osteoclast number in long bones. However, osteoclast numbers were increased in their femur in response to unloading (29) and intermittent PTH (see below), as well as in alveolar bone (jaw) (20). Moreover, wild type primary osteoclasts cultured with primary osteoblasts from Postn KO mice show a higher number and activity of osteoclasts as compared to co-cultures with wild-type osteoblasts. In turn adding rPostn to osteoclastic cultures decreased the number of osteoclasts, as well as their activity (30).

Recently mice deficient for Wnt16 have revealed the unique functions of this molecule on the skeleton as these mice were characterized by decreased cortical, but not trabecular, bone mass 
and strength. Similar to Postn KO mice, Wnt16 KO mice present decrease in bone formation and mechanical responses at the periosteum and increased bone resorption endocortically (REF). Interestingly, in these mice, Postn expression is massively down-regulated (31).

Finally, Postn $\mathrm{KO}$ mice also exhibit some alterations in bone material properties, including reduced collagen cross-linking with an enlargement of collagen fibers, lower levels of mineralization, and increased levels of microdamage (32). The orientation of osteocytic lacunae, which normally appears in concentric layers parallel to the cortical bone surface, was also disorganized (Figure 3).

\section{Roles of periostin in the bone biomechanical responses}

In wild type mice, axial compression of the tibia increases Postn mRNA and protein levels in bone (28). These changes are both spatially and temporally related to the inhibition of Sost expression and the increase in bone forming indices. Interestingly, periostin is preferentially expressed in the outer layers of the cortex and close to surfaces exhibiting the greatest increase in bone formation (28). These findings suggest that periostin may serve as a molecular conduit to concentrate biomechanical signals to the outer surfaces of bone, where gains in mass would have the most favorable impact on bone strength. Accordingly, the cortical bone response to both axial compression and treadmill exercise was markedly blunted in Postn KO mice. In these mice, Sost was overexpressed and was not inhibited following loading. In turn, sclerostin neutralizing Ab partially rescued the biomechanical response to loading in Postn $\mathrm{KO}$ mice. These results argue for an additional role of periostin on osteoblast independently of sclerostin signaling pathway.

Three other publications independently confirmed the role of Postn in response to exercise and its interaction with sclerostin expression. The first one confirmed an increase of periostin expression in osteoblasts and osteocytes in response to 3 to 12 weeks of intense exercise, 
which is a model of work-related musculoskeletal overload (33). The second showed that periosteal bone formation and improved bone microarchitecture in response to exercise was abolished in mice deficient for the microRNA miR17-92, in which the expression of Elk3, Runx2 and Postn remained low (34). The last one demonstrated that alteration of the skeleton and lower alveolar bone volumes in Postn $\mathrm{KO}$ mice were attributed to an overexpression of sclerostin. Moreover, by crossing Postn $\mathrm{KO}$ with Sost $\mathrm{KO}$ mice, the altered alveolar bone phenotype of the former was normalized (35). Altogether these experiments demonstrate that Postn expression and its induction by mechanical forces occur upstream of sclerostin inhibition in the bone mechanostatic pathway. To confirm that periostin mediates the bone biomechanical response through Wnt- $\beta$ catenin $(\mathrm{Ctnn} \beta)$ activation within the osteocyte, osteocyte-specific Ctnn $\beta 1$ / Postn double haploinsufficient mice were generated. In these mice, the cortical bone response to mechanical stimulation was altered to a similar degree as in Postn $\mathrm{KO}$ mice (36). More recently the interactions between periostin and the sclerostinWnt/ $\beta$-catenin signaling pathway has been confirmed and targeted as strategy to prevent neurectomy-induced bone loss (37).

Considering the functions of periostin on bone matrix structure and material properties (above), as well as on osteocytes functions, it was expected to also be involved in bone resistance to stress fractures and injury repair. Upon fatigue loading of the tibia, Postn KO mice presented more cracks than wild-type littermate, and these cracks persisted longer, arguing for some additional functions of periostin in the damage remodeling process. This is likely to occur through the stimulation of OPG expression by the activation of $\beta$-catenin signaling in osteocytes (see above) (3). Moreover, and consistent with periostin role on modeling-based bone formation, the callus bone volume in response to fatigue loading was diminished in Postn KO mice, resulting in lower bone strength (32). 
There is also some evidence that periostin plays a role during mechanical unloading, a process known to induce bone loss through an unbalanced process of increased bone resorption and decreased bone formation $(38,39)$. Hence tail-suspension results in deterioration of trabecular and cortical structures as well as suppression of periosteal and endocortical bone formation. These changes are accompanied by an early and persistent downregulation of Postn expression and a concomitant upregulation of Sost (at 7 days), followed by Dkk1 (at 21 days), whereas the Rankl/Opg expression ratio increases progressively. Consistent with a role for Postn in the regulation of Sost (see above), Sost mRNA levels do not significantly increase in response to unloading in Postn deficient mice (40). In contrast, Dkk1 increases similarly to wild type, and changes in Opg and RankL are also unaffected, indicating that in these conditions, Postn selectively regulates Sost expression in osteocytes. As a result, low levels of periosteal bone formation rate were maintained in tail-suspended Postn $\mathrm{KO}$ mice, which did not loose further cortical bone mass. In contrast, trabecular bone loss in response to unloading persisted even in the absence of Postn, again supporting the specific role of this molecule on cortical bone homeostasis.

\section{Role of periostin in response to intermittent PTH}

Postn expression is highly upregulated by PTH in human osteoblasts, in mice, as well as in bone extracts from patients with primary hyperparathyroidism (41-45). Serum periostin levels are also significantly increased in response to intermittent PTH (iPTH) in mice, and correlate with cortical structure parameters, independently of P1NP and TRAP (Figure 4A-B). In Postn $\mathrm{KO}$ mice, iPTH did not increase cortical bone forming indices nor cortical bone volume or thickness (18). In contrast, PTH-stimulated bone formation on trabecular surfaces was maintained in absence of Postn. Hence, absence of Postn leads to compartment-specific alterations in the response to iPTH. 
Similar to mechanical loading, the bone forming effects of iPTH appear to be at least partially mediated through the inhibition of sclerostin. PTH-stimulated increases in bone mass and structure are blunted in mice over-expressing Sost, as well as in Sost-deficient mice $(46,47)$. Sclerostin protein expression is not suppressed by $\mathrm{iPTH}$ in the bones of Postn $\mathrm{KO}$ mice. Consistent with these in vivo observations, in UMR-106 osteoblast-like cells, periostin neutralizing antibodies partially prevented the PTH-induced downregulation of Sost and its transcription factor Mef2C (18). However, sclerostin-blocking antibodies do not fully restore iPTH effects in Postn KO mice, which differs from the full rescue observed in these animals when subjected to mechanical loading (28). These observations further suggest that PTHstimulated Postn expression may exert its effects on cortical bone formation not only by down-regulating Sost but also by directly stimulating lining cells. Consistent with this hypothesis, the PTH-stimulated activation of $\beta$-catenin signaling observed in osteocytes and osteoblasts at the endocortical and periosteum surfaces using TOPGAL reporter mice was virtually suppressed in Postn KO mice (18). These results identify periostin as a regulator of the Sost/Wnt/ $\beta$-catenin signaling pathway not only in response to mechanical loading but also in the anabolic response to PTH. Finally, periostin can regulate bone formation through a direct stimulation on Wnt $\beta$ catenin, and via indirect stimulation of Wnt $\beta$ catenin through sclerostin inhibition, as well as through non-canonical Wnt signaling pathways, as also previously demonstrated in epithelial cells (48).

\section{Periostin as a potential clinical biomarker}

Recently two commercially available immunoassays have been developed to measure circulating periostin. The assay from USCN (China), uses a polyclonal antibody raised against the FAS-1 domain of Postn and is expected to detect all Postn isforms. A second more recent ELISA from Adipogen (Switzerland) uses two monoclonal antibodies of unknown specificity 
and it remains unclear which molecular forms it detects. Using these ELISA, it has been shown that in postmenopausal women serum periostin did not (49-51) or only weakly (52) correlate with spine and hip areal BMD . Similarly serum Postn was not or only slightly associated with conventional markers of bone formation and bone resorption in the same clinical studies. These data indicate that when assayed in serum, Postn levels reflect biological processes which are different from those captured by static (BMD) or dynamic (bone markers) indices of bone metabolism. $(50,51)$. In a cohort of healthy elderly men and women, a positive correlation between serum periostin and QCT assessed cortical bone thickness was observed whereas it correlated negatively with cortical porosity. In the same individuals, serum periostin was not correlated with trabecular bone volume fraction (BV/TV) after adjustment by P1NP, CTX, BMD, gender or age (51). There was also an association of periostin levels between parents and adult offspring, with an estimated heritability of $50 \%$, and a genetic covariance was found between serum periostin and bone microarchitecture. In a cohort of post-menopausal women, the highest quartile of serum periostin measured by the USCN assay was associated with an increased risk of incident all fractures, independently of BMD and conventional bone markers (50). These data were more recently confirmed in another case control study of postmenopausal women from Korean, in which serum periostin measured by the Adipogen ELISA was also positively associated with incident fracture independently of BMD (52). Interestingly, when vertebral and non-vertebral fractures were analysed separately, periostin levels were associated only with the latter fracture type, which is in agreement with the predominant role of Postn on cortical bone metabolism. These clinical data suggest that serum periostin could be a new biological marker of fracture risk, and more specifically non vertebral fracture in postmenopausal women. The fact that it is independent of both BMD and bone markers suggests that the measurement of serum periostin could be useful in combination with these two key risk factors to improve risk 
assessment. These data need however to be confirmed in larger independent studies in which these hypotheses can be tested. It has however to be remembered that periostin is not specific of bone and that serum levels represent the additional contribution of the metabolism of other tissues. It is possible that bone specific isoforms or Postn fragments could be a more sensitive index of fracture risk. In that respect, it is interesting to note that specific periostin isoforms have been described in muscle (53) and periodontal ligament (54).

\section{Summary and perspectives}

Experiments in vitro and in Postn $\mathrm{KO}$ mice have started to delineate the multiple roles of periostin on bone homeostasis, particularly in the bone modeling response to mechanical stimuli and PTH through the regulation of sclerostin expression and the WNT- $\beta$ catenin signaling pathway in osteoblasts and osteocytes. Through the latter, periostin could also modulate bone remodeling by regulating the expression of OPG, which is particularly important in damage repair. Since serum periostin levels appear to decrease with age and to be lower in women, we postulate that down-regulation of Postn expression could be a mechanism to explain the accumulation of microcracks with age and more so in women (55), as well as the declining response to exercise in the aging skeleton. On another side, increased periostin expression could play a relatively protective role on bone mass in hyperparathyroidism and its preferential expression in the outer cortex contribute to the uneven distribution of cortical porosity in this disease. Furthermore, periostin appears to be degraded by cysteine proteases such as Cathepsin K, suggesting that its level of expression could be modulated by Cathepsin $\mathrm{K}$ inhibitors and thereby play a role in the maintenance of bone forming surfaces observed with certain Cathepsin K pharmacological inhibitors, such as odanacatib $(56,57)$. Eventually, the functions of periostin as a bone matrix constituent and the 
alterations of bone material properties observed in Postn KO mice could suggest a role of low periostin levels in fatigue and atypical femoral fractures, which remains to be investigated.

\section{Figure Legends}

Figure 1. Signaling pathways of periostin, adapted from Morra et al (12). Periostin binding to integrins initiates integrins signaling and promotes the recruitment of tyrosine kinases receptor (RTK) at the plasma membrane. This crosstalk activates Ras/MAPK/ERK, Akt/PKB and the FAK mediated signaling pathway. It is important to point-out that the control of GSK$3 \beta$ phosphorylation by Akt is more and more highlighted as an important regulator of $\beta$ catenin $(\beta C \operatorname{ctn})$ signalling pathways. The convergence of these pathways results in the activation of select transcription factors such Notch1, $\beta$-catenin..., most important pathway in osteoblast / osteocyte; which control cell survival, proliferation, migration, differentiation and apoptosis. Mitogen-activated protein kinase (MAPK); Focal adhesion kinase (FAK); phosphatidylinositol (3,4,5)-trisphosphate (PIP3); Interleukin kinase (ILK); mammalian target of rapamycin (mTOR).

Figure 2. Periostin expression in IDG-W3 (for Immortomouse/Dmp1-GFP-SW3) late osteoblast/osteocyte. (A) Relative expression of periostin along osteoblast differentiation into osteocyte which can be followed in live through GFP. (B) Immunostainning of periostin in IDG-W3 at days 21 after 24 hours of vehicle (Veh) or PTH.

Figure 3. Illustration of bone shape in absence of periostin with aging. A. Under normal aging despite periosteal apposition exceed endocortical resorption and therefore the external diameter increase, the cortex become thicker due to an increase in endo and intra-cortical resorption. It is interesting to note that bone formation is note uniform all around the bone but 
follow the mechanical distribution with a high strain on the antero-lateral surface vs posteromedial surface. In absence of periostin, double calcein injection indicates a decrease in mineral apposition rate and lower surface of labeling. Moreover, we can detect an increase in intra-cortical remodeling as indicated by calcein labeling in the pore indicating a role of periostin not only in modeling activity (indicate by arrow). We also report a disorganized osteocyte lacunae in the third external part of the cortex (indicate by $\$$ ). Thereby the most striking phenotype is a small outer diameter with a low Ct.BV and Ct.Th vs wild-type.

Figure 4. Correlation of sPOSTN with bone formation indices and cortical bone microarchitecture parameters, independently of bone turnover markers. (A) Five weeks of intermittent PTH significantly increased sPOSTN levels, *p<0.05 vs baseline; (B) Better correlation of sPOSTN with bone formation indices and cortical microarchitecture than P1NP.

\section{Acknowledgments}

We thank Dr. Conway (Program in Developmental Biology and Neonatal Medicine, Wells Center for Pediatric Research, Indiana University School of Medicine, Indianapolis, USA) for providing the periostin deficient mice. We also thank Pr Lynda Bonewald (University of Missouri, Kansas, USA) for providing the IDG-W3 cell lines. We thank Ms Madelein Lachize Julia Brun and Juliette Zicchinni for her technical assistance. Our investigation on periostin were supported by a Swiss National Science Foundation grant No 3100A0-116633/1 (to S.L.F); and by a grant from the ECTS-Amgen fellowship (to N.B).

\section{References}

1. Bonewald LF. The amazing osteocyte. J Bone Miner Res 2011;26(2):229-38. 
2. Baron R, Kneissel M. WNT signaling in bone homeostasis and disease: from human mutations to treatments. Nat Med 2013;19(2):179-92.

3. Kramer I, Halleux C, Keller H, Pegurri M, Gooi JH, Weber PB, et al. Osteocyte Wnt/beta-catenin signaling is required for normal bone homeostasis. Mol Cell Biol. 2010;30(12):3071-85.

4. Takeshita S, Kikuno R, Tezuka K, Amann E. Osteoblast-specific factor 2: cloning of a putative bone adhesion protein with homology with the insect protein fasciclin I. Biochem $\mathbf{J}$ 1993;294 271-8.

5. Merle B, Garnero P. The multiple facets of periostin in bone metabolism. Osteoporos Int. 2012;23(4):1199-212.

6. Coutu DL, Wu JH, Monette A, Rivard GE, Blostein MD, Galipeau J. Periostin, a member of a novel family of vitamin K-dependent proteins, is expressed by mesenchymal stromal cells. J Biol Chem. 2008;283(26):17991-8001.

7. Kudo A. Periostin in fibrillogenesis for tissue regeneration: periostin actions inside and outside the cell. Cell Mol Life Sci. 2011;68(19):3201-7.

8. Gillan L, Matei D, Fishman DA, Gerbin CS, Karlan BY, Chang DD. Periostin secreted by epithelial ovarian carcinoma is a ligand for alpha(V)beta(3) and alpha(V)beta(5) integrins and promotes cell motility. Cancer Res 2002;62(18):5358-64.

9. Shimazaki M, Nakamura K, Kii I, Kashima T, Amizuka N, Li M, et al. Periostin is essential for cardiac healing after acute myocardial infarction. J Exp Med 2008;205(2):295303.

10. Ruan K, Bao S, Ouyang G. The multifaceted role of periostin in tumorigenesis. Cell Mol Life Sci 2009;66(14):2219-30.

11. Ghatak S, Misra S, Norris RA, Moreno-Rodriguez RA, Hoffman S, Levine RA, et al. Periostin induces intracellular cross-talk between kinases and hyaluronan in atrioventricular valvulogenesis. J Biol Chem. 2014;289(12):8545-61.

12. Morra L, Moch H. Periostin expression and epithelial-mesenchymal transition in cancer: a review and an update. Virchows Arch 2011;459(5):465-75.

13. Tkatchenko TV, Moreno-Rodriguez RA, Conway SJ, Molkentin JD, Markwald RR, Tkatchenko AV. Lack of periostin leads to suppression of Notch1 signaling and calcific aortic valve disease. Physiol Genomics 2009;39(3):160-8.

14. Rosselli-Murai LK, Almeida LO, Zagni C, Galindo-Moreno P, Padial-Molina M, Volk SL, et al. Periostin responds to mechanical stress and tension by activating the MTOR signaling pathway. PLoS One. 2013;8(12):e83580.

15. Horiuchi K, Amizuka N, Takeshita S, Takamatsu H, Katsuura M, Ozawa H, et al. Identification and characterization of a novel protein, periostin, with restricted expression to periosteum and periodontal ligament and increased expression by transforming growth factor beta. J Bone Miner Res 1999;14(7):1239-49.

16. Zhu S, Barbe MF, Liu C, Hadjiargyrou M, Popoff SN, Rani S, et al. Periostin-likefactor in osteogenesis. J Cell Physiol 2009;218(3):584-92.

17. Litvin J, Selim AH, Montgomery MO, Lehmann K, Rico MC, Devlin H, et al. Expression and function of periostin-isoforms in bone. J Cell Biochem 2004 92(5):1044-61.

18. Bonnet N, Conway SJ, Ferrari SL. Regulation of beta catenin signaling and parathyroid hormone anabolic effects in bone by the matricellular protein periostin. Proc Natl Acad Sci U S A. 2012;109(37):15048-53.

19. Chan CSL, Leung CM, Chan D, Cheah KSE, Tanner JA Periostin interacts with sclerostin and inhibits its antagonistic effect on Wnt signalling. International Bone and Mineral Workshops. 2008; Switzerland March 9-11:Davos.

20. Bonnet N, Lesclous P, Saffar JL, Ferrari S. Zoledronate effects on systemic and jaw osteopenias in ovariectomized periostin-deficient mice. PLoS One. 2013;8(3):e58726. 
21. Merle B, Bouet G, Rousseau JC, Bertholon C, Garnero P. Periostin and transforming growth factor $\beta$-induced protein (TGF $\beta I$ ) are both expressed by osteoblasts and osteoclasts. Cell Biol Int. 2014;38(3):398-404.

22. Heo SC, Shin WC, Lee MJ, Kim BR, Jang IH, Choi EJ, et al. Periostin accelerates bone healing mediated by human mesenchymal stem cell-embedded hydroxyapatite/tricalcium phosphate scaffold. PLoS One. 2015;10(3):e0116698.

23. Thorfve A, Bergstrand A, Ekström K, Lindahl A, Thomsen P, Larsson A, et al. Gene expression profiling of peri-implant healing of PLGA-Li+ implants suggests an activated Wnt signaling pathway in vivo. PLoS One. 2014;9(7):e102597.

24. Zhang Z, Nie F, Chen X, Qin Z, Kang C, Chen B, et al. Upregulated periostin promotes angiogenesis in keloids through activation of the ERK 1/2 and focal adhesion kinase pathways, as well as the upregulated expression of VEGF and angiopoietin-1. Mol Med Rep. 2015;11(2):857-64.

25. Rios H, Koushik SV, Wang H, Wang J, Zhou HM, Lindsley A, et al. periostin null mice exhibit dwarfism, incisor enamel defects, and an early-onset periodontal disease-like phenotype. Mol Cell Biol. 2005;25(24):11131-44.

26. Wilde J, Yokozeki M, Terai K, Kudo A, Moriyama K. The divergent expression of periostin mRNA in the periodontal ligament during experimental tooth movement. Cell Tissue Res. 2003;312(3):345-51.

27. Kii I, Amizuka N, Minqi L, Kitajima S, Saga Y, Kudo A. Periostin is an extracellular matrix protein required for eruption of incisors in mice. Biochem Biophys Res Commun. 2006;342(3):766-72.

28. Bonnet N, Standley KN, Bianchi EN, Stadelmann V, Foti M, Conway SJ, et al. The matricellular protein Periostin is required for Sclerostin inhibition and the anabolic response to mechanical loading and physical activity. J Biol Chem 2009;284(51):35939-50.

29. Gerbaix M, Vico L, Ferrari SL, Bonnet N. Periostin expression contributes to cortical bone loss during unloading. Bone. 2015;71:94-100.

30. Bonnet N, Ferrari S. Periostin prevents cortical bone loss by increasing OPG levels in response to continuous PTH in mice. J Bone Min Res. 2015;s24.

31. Wergedal JE, Kesavan C, Brommage R, Das S, Mohan S. Role of WNT16 in the regulation of periosteal bone formation in female mice. Endocrinology. 2015;156(3):1023-32.

32. Bonnet N, Gineyts E, Ammann P, Conway SJ, Garnero P, Ferrari S. Periostin deficiency increases bone damage and impairs injury response to fatigue loading in adult mice. PLoS One. 2013;8(10):e78347.

33. Rani S, Barbe MF, Barr AE, Litvin J. Periostin-like-factor and Periostin in an animal model of work-related musculoskeletal disorder. Bone. 2009;44(3):502-12.

34. Mohan S, Wergedal JE, Das S, Kesavan C. Conditional disruption of miR17-92 cluster in collagen type I-producing osteoblasts results in reduced periosteal bone formation and bone anabolic response to exercise. Physiol Genomics. 2015;47(2):33-43.

35. Ren Y, Han X, Ho SP, Harris SE, Cao Z, Economides AN, et al. Removal of SOST or blocking its product sclerostin rescues defects in the periodontitis mouse model. FASEB J. 2015; In press.

36. Bonnet N, Ferrari S. Periostin (Postn) synergizes with osteocytes beta-catenin to mediate the adaptive skeletal response to loading Bone. 2013;47:ECTS meeting.

37. Lv J, Sun X, Ma J, Ma X, Xing G, Wang Y, et al. Involvement of periostin-sclerostin$\mathrm{Wnt} / \beta$-catenin signaling pathway in the prevention of neurectomy-induced bone loss by naringin. Biochem Biophys Res Commun. 2015; In press.

38. Vico L, Alexandre C. Microgravity and bone adaptation at the tissue level. J Bone Miner Res. 1992;7(Suppl 2):S445-7. 
39. Vico L, Lafage-Proust MH, Alexandre C. Effects of gravitational changes on the bone system in vitro and in vivo. Bone. 1998;22(5 Suppl):95S-100S.

40. Gerbaix M, Vico L, Ferrari SL, Bonnet N. Periostin expression contributes to cortical bone loss during unloading. Bone. 2015;71:94-100.

41. Bianchi EN, Ferrari SL. Beta-arrestin2 regulates parathyroid hormone effects on a p38 MAPK and NFkappaB gene expression network in osteoblasts. Bone 2009;45(4):716-25.

42. Onyia JE, Helvering LM, Gelbert L, Wei T, Huang S, Chen P, et al. Molecular profile of catabolic versus anabolic treatment regimens of parathyroid hormone (PTH) in rat bone: an analysis by DNA microarray. J Cell Biochem. 2005;95(2):403-18.

43. Li X, Liu H, Qin L, Tamasi J, Bergenstock M, Shapses S, et al. Determination of dual effects of parathyroid hormone on skeletal gene expression in vivo by microarray and network analysis. J Biol Chem. 2007;282(45):33086-97.

44. Reppe S, Stilgren L, Olstad OK, Brixen K, Nissen-Meyer LS, Gautvik KM, et al. Gene expression profiles give insight into the molecular pathology of bone in primary hyperparathyroidism. Bone. 2006;39(1):189-98.

45. Fortunati D, Reppe S, Fjeldheim AK, Nielsen M, Gautvik VT, Gautvik KM. Periostin is a collagen associated bone matrix protein regulated by parathyroid hormone. Matrix Biol. 2010;29(7):594-601.

46. Robling AG, Kedlaya R, Ellis SN, Childress PJ, Bidwell JP, Bellido T, et al. Anabolic and catabolic regimens of human parathyroid hormone 1-34 elicit bone- and envelope-specific attenuation of skeletal effects in Sost-deficient mice. Endocrinology. 2011;152(8):2963-75.

47. Kramer I, Keller H, Leupin O, Kneissel M. Does osteocytic SOST suppression mediate PTH bone anabolism? Trends Endocrinol Metab 2010;21(4):237-44.

48. Haertel-Wiesmann M, Liang Y, Fantl WJ, Williams LT. Regulation of cyclooxygenase- 2 and periostin by Wnt-3 in mouse mammary epithelial cells. J Biol Chem. 2000;275(41):32046-51.

49. Anastasilakis AD, Polyzos SA, Makras P, Savvides M, Sakellariou GT, Gkiomisi A, et al. Circulating periostin levels do not differ between postmenopausal women with normal and low bone mass and are not affected by zoledronic acid treatment. Horm Metab Res. 2014;46:145-9.

50. Rousseau J, Sornay-Rendu E, Bertholon C, Chapurlat R, Garnero P. Serum periostin is associated with fracture risk in postmenopausal women: a 7 years prospective analysis of the OFELY study. J Clin Endocrinol Metab 2014;3893:1-7.

51. Bonnet N, Biver E, Durosier C, Chevalley T, Rizzoli R, Ferrari S. Additive genetic effects on circulating periostin contribute to the heritability of bone microstructure. J Clin Endocrinol Metab 2015;100(7):E1014-21.

52. Kim BJ, Rhee Y, Kim CH, Baek KH, Min YK, Kim DY, et al. Plasma periostin associates significantly with non-vertebral but not vertebral fractures in postmenopausal women: Clinical evidence for the different effects of periostin depending on the skeletal site. Bone. 2015;81:435-41.

53. Ozdemir C, Akpulat U, Sharafi P, Yıldız Y, Onbaşılar I, Kocaefe C. Periostin is temporally expressed as an extracellular matrix component in skeletal muscle regeneration and differentiation. Gene. 2014;553(2):130-9.

54. Yamada S, Tauchi T, Awata T, Maeda K, Kajikawa T, Yanagita M, et al. Characterization of a novel periodontal ligament-specific periostin isoform. J Dent Res. 2014;93(9):891-7.

55. Schaffler MB, Choi K, Milgrom C. Aging and matrix microdamage accumulation in human compact bone. Bone. 1995;17(6):521-25.

56. Duong le T. Therapeutic inhibition of cathepsin K-reducing bone resorption while maintaining bone formation. Bonekey Rep. 2012;2(1):67. 
57. Lotinun S, Kiviranta R, Matsubara T, Alzate JA, Neff L, Lüth A, et al. Osteoclastspecific cathepsin $\mathrm{K}$ deletion stimulates S1P-dependent bone formation. J Clin Invest 2013;123(2):666-81. 
Circulating levels are higher in men than in women

Linked to fibronectin, tenascin-C and collagen 1

Expressed in lining cells, osteoblasts and osteocytes

Responsive to PTH, TGF- $\beta$ and mechanical loading

Binding to $\alpha \mathrm{V} \beta 3$ integrin receptors

Signaling through AKT/FAK and Wnt-beta-catenin pathways

Down-regulates Sost

May upregulate OPG

Effects on osteoblasts migration, proliferation and differentiation

Effects on osteocytes apoptosis

Direct effects on osteoclasts remains to be investigated
At the bone tissue level there are no specific sex difference in Posth $^{-/-}$mice

$\downarrow$ osteoblast proliferation, mineralization and attachment on bone matrix

$\downarrow \downarrow$ femoral BMD

$\downarrow \downarrow$ cortical bone volume and thickness

$\downarrow$ trabecular bone volume on tissue volume

$\downarrow$ bone formation (MAR) mainly at the periosteal surface

Altered collagen organization and $\downarrow$ levels of crosslinks (PYD/DPD, DHLNL / HLNL)

$\uparrow$ microcracks accumulation on repair ratio

Alter osteocytic network organization

$\downarrow$ extrinsic bone strength and intrinsic mechanical properties (Hardness)

Table 1. Functions of periostin in bone homeostasis. Immature crosslinks (dihydroxylysinonorleucine, DHLNL and hydroxylysinonorleucine, HLNL) and mature crosslinks (pyridinoline, PYD and deoxypyridinoline, DPD) in bone extracts. 


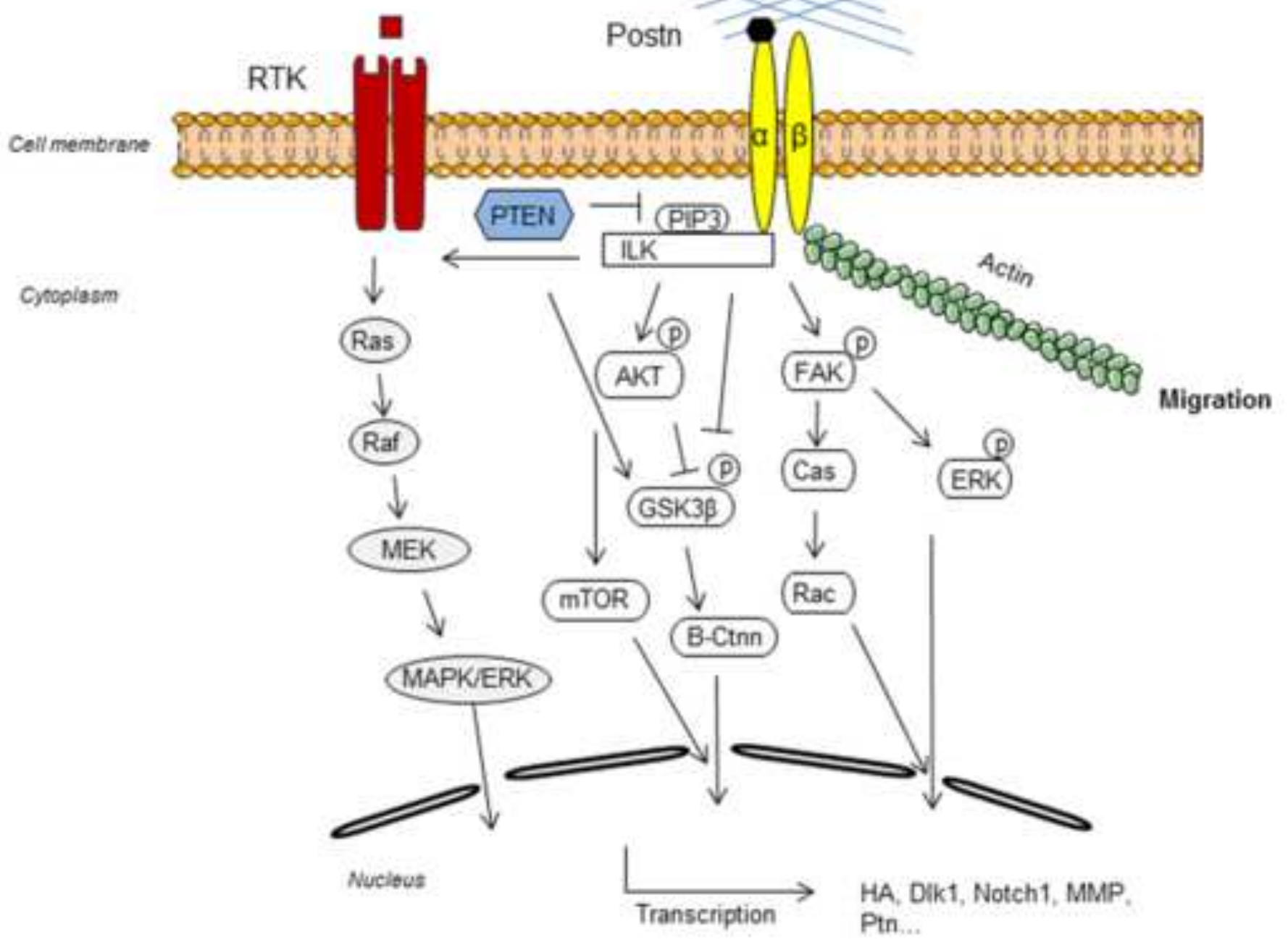

Fig. 1 
A
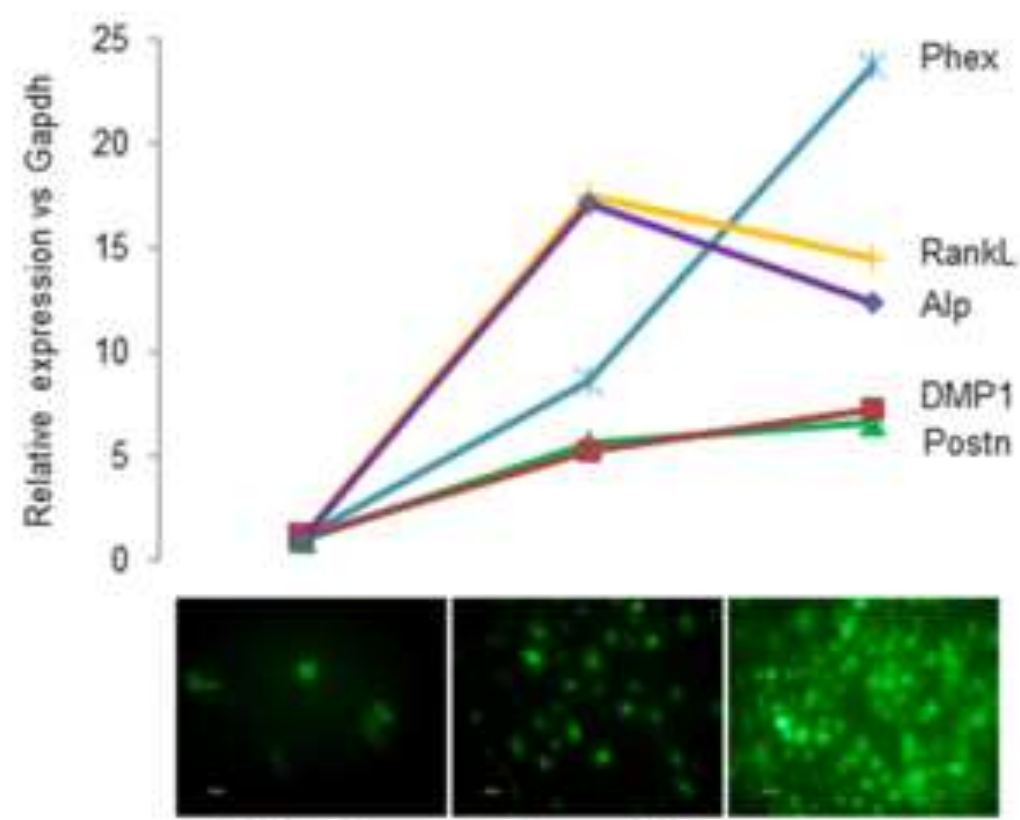

Days 4

Days 10

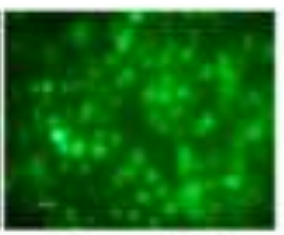

Days 21

B
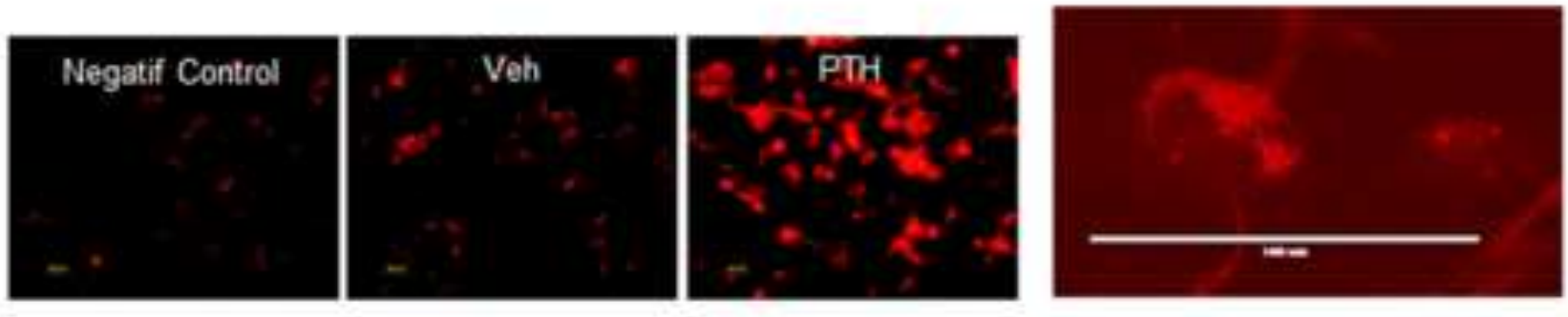

Fig. 2

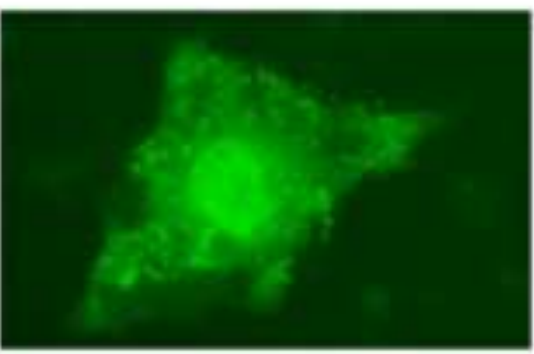




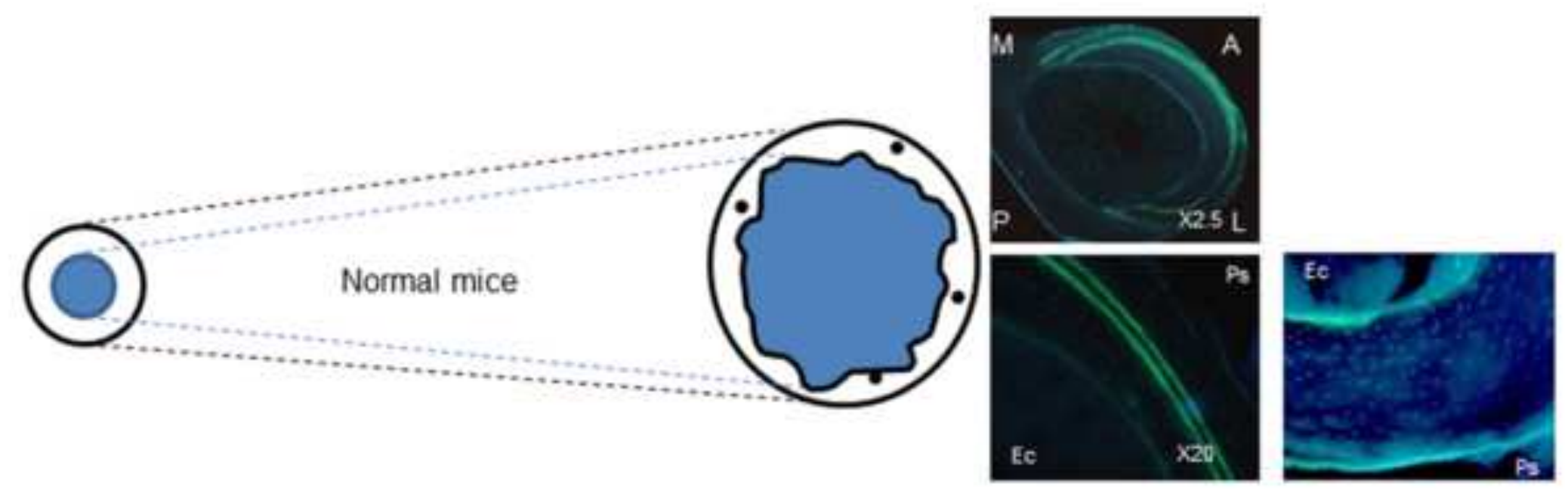

With Age

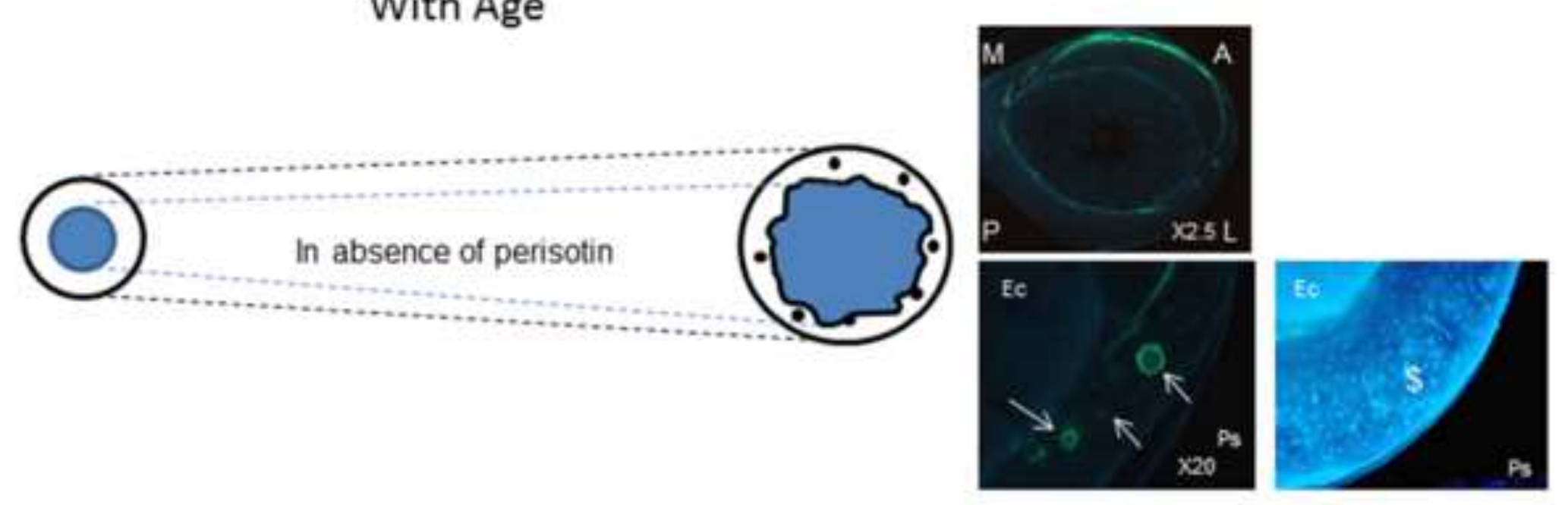

Fig. 3 
A

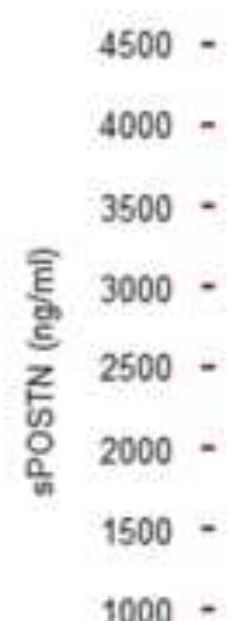$$
-\int_{1}^{\infty}
$$$$
\text { }
$$$$
\text { Time }
$$$$
\leadsto \text { 5Week }
$$$$
\text { - Gase }
$$

B

$$
\text { Ec }
$$

Ps

\begin{tabular}{c|l|l|l|l|l|l|l|l|l|c}
$r^{2}$ & TRAP & P1NP & MAR & MPm/BPm & BFR/ BPm & MAR & MPm/BPm & BFR/ BPm & Ct.BV & MI \\
\hline sPOSTN & 0.11 & 0.01 & $0.43 \cdots$ & $0.28 *$ & $0.32 *$ & $0.31 *$ & $0.39 \cdots$ & $0.33^{*}$ & $0.30^{*}$ & $0.24 *$ \\
\hline P1NP & 0.01 & 1 & 0.12 & $0.28 *$ & $0.24^{*}$ & 0.07 & 0.17 & 0.14 & 0.025 & 0.09 \\
\hline
\end{tabular}

Fig. 4 